\title{
Sound Bending - Talking Bodies Quantum Sound Suits
}

\author{
Kristin Neidlinger ${ }^{1}$ and Wendy $\mathrm{Ju}^{2}$ \\ ${ }^{1}$ SENSOREE Therapuetic bio.media, \\ 2069a Mission Street, \\ San Francisco, CA 94122 \\ ${ }^{2}$ Center for Design Research, \\ 424 Panama Mall, Bldg 560, \\ Stanford, CA 94306 \\ kristinasensoree.com, wendyjuastanford.edu
}

\begin{abstract}
The QuantumSound Suits are an innovative technological solution for creating sounds from movement. Made in collaboration with contortionists, a multidisciplinary team designed custom body-painted silicone suits embedded with flexible sensors. A healing sound artist mapped the tones of the eleven sensors to movement, animating the physical motion and providing sonic feedback of body's position. This is an exploration of real-time movement notation and human activity recognition of body location in space.
\end{abstract}

Keywords: human activity recognition, wearables, sonification, performance, Arduino, dance, transdiscliplinary collaboration.

\section{Introduction}

QuantumSound bodysuits create sounds from movement. These custom molded silicone suits have a network of flexible sensors at their joints. As the contortionists move, a sound-scape is created that is designed to align the body mind, and lights glow to draw attention to key aspects of the movement.

The custom molded silicone suits of QuantumSound were created as part of a collaborative design process. Working with a contortion duet, we iteratively designed movements that were both expressive for performance and capable of being sensed using wearable sensors. The locations of the sensors were used by a healing sound artist to create tones intended to help the alignment of the body mind. Finally, the wearable sensors and the accompanying electronics were integrated into suits for each dancer. In this work, we will fully describe the design process of QuantumSound, detail the overall architecture of the final system, and reflect on lessons from our project.

\section{Related Work}

Laurie Anderson was among the first to illustrate the performative potential of the instrumented body in the 1980s; in her Drum Dance, she donned an electronic body 
suit which allowed her to trigger percussive samples when she drummed on different parts of her body. [1] The suit makes the dancer's body the "ultimate portable instrument," as Anderson put it [2], collapsing the set, the props, the musical accompaniment and the visual performance onto the body and movement of the dancer.

Troika Ranch's MidiDancer similarly used bodysuits to augment dance performance, but instead of using piezo elements that recognized taps, the MidiDancer bodysuits incorporated plastic fibers that responded to the flexion and extension of the major joints on the body [3]. These inputs are then used to modify the projected visual and auditory stage surrounding the performers. Whereas Anderson's Drum Dance featured Anderson "playing" her body, tapping different body parts to trigger different sounds, the Troika Ranch dancer was given second-order control over the sound: the performer might, for example, be able to amplify a sound from soft to loud by curling an extended arm.

The early instances dance performance technologies started in the late 1990's to snowball into a wider-array of "wearable computers" intended to augment performance. Articles of augmented clothing such as instrumented tracksuits [4], and dancer's shoes [5] were outfitted with accelerometers, gyros, bend sensors, buttons and switches, as well as wireless communication, to enable both sonification of movement and higher level control of sound and video. Mulder [6] and Winkler [7] introduced taxonomies to help classify these within a wider array of human movement-tracking technologies; these "inside-in" [6] systems utilize "body sensors" [7] to track sources on the body, but do not locate the body relative to external points such as placement on the stage.

Siegel and Jacobsen, in their 1998 article on the challenges of interactive dance, note that inside-in systems often restrict movement and are often aesthetically obtrusive. [8] In addition, striking the right balance between overly simple one-to-one mappings of movement to sound and more complex relationships that are richer but harder for the audience to perceive, is an obstacle. Finally, they note that the nature of these interactive technologies changes the relationship of the choreographer, dancer and composer.

The maturation of technologies and collaboration processes brings new possibilities to the realm of interactive dance performance. The sensing, computing and communication technologies have become lighter weight, lower in cost, and easier to develop, allowing for greater access, flexibility and experimentation. In addition, the intervening years have also changed the nature of collaborative interactions. Whereas in Anderson's day the technologies really centered around the dancer as the performer-artist, and in Seigel and Jacobsen's era, the blurring of the lines between dancer, composer and choreographer was felt to post great challenges for the dancer, at this point in time, the increasingly transdisciplinary and iterative nature of artistic collaborations has become more normative. 


\section{Project Overview}

QuantumSound was created as part of a residency at the Crucible foundry in Oakland, which culminated in a Maker's Fashion Show [9] in January 2013. The organizing staff at the Crucible chose twelve designers to be part of the residency program, which lasted two months. The requirement was to already have ideas and a team. Our design team for this piece was comprised of seven members: a software engineer, a silicone artist, a sound designer, a hardware engineer, the two contortionists, and the design lead. The team was assembled at the start of the project.

\subsection{Motivation/Objective}

The QuantumSound performance was primarily driven by a desire to make a soundmovement costume that animated the body. The lead designer was inspired by observing a performer whose movements seemed to have an inherent musicality to them, and a desire to hear the music of those movements as they were made in the space. She was also inspired to create a sound game with sound rewards for effort. Sound and light would reward proper body positions, and motivate the dancers towards moving in specific ways.

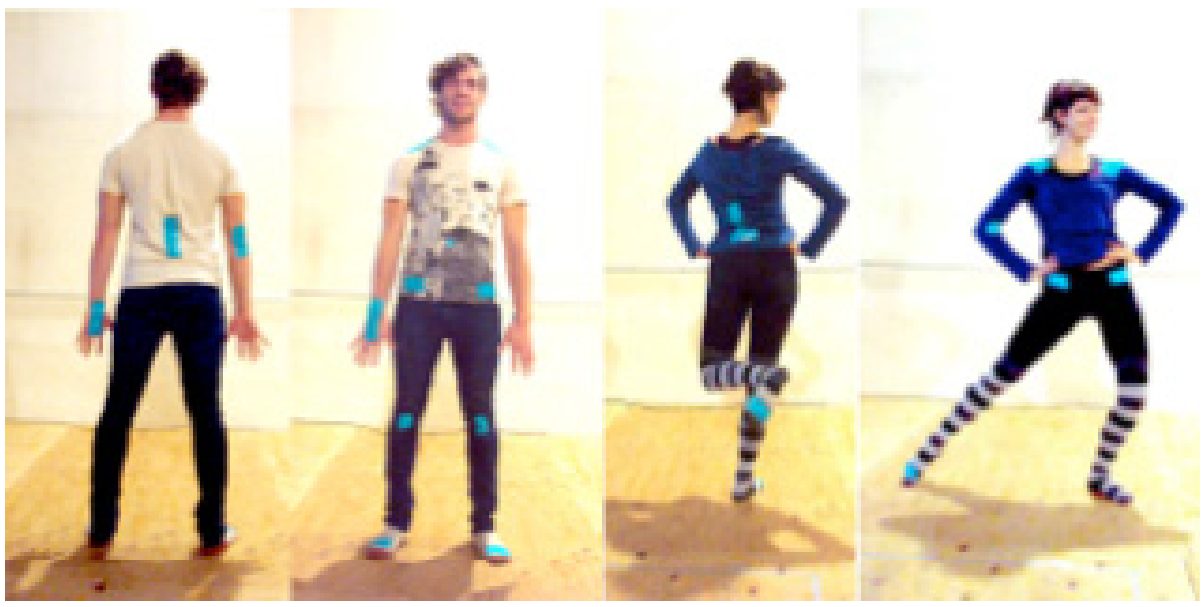

Fig. 1. Selected movement points on body chosen by contortionists drove sensor selection and placement

\subsection{Process}

SENSOREE collaborated with two contortionists to design the suits over the course of ten sessions of roughly two hours each, in residency at the contortionists' training gym. The sensor design, the physical fit of the wearable sensors, and the sound design all occurred iteratively during these sessions. 


\subsection{Mapping Movements and Sensing Points}

Collectively, we selected specific movements and choreography where we imagined sound would be illuminating. The placement of the sensors was chosen by the contortionists based on their movement choreography preferences. We started with the material that they had developed, in solo preferences and in duets and found locations on the body that animated their personal movements.

Examining the movement, we then worked on ways to instrument the contortionists' bodies to sonify that movement. For instance, Elliott prefers hand balancing, so on the wrists and palms, we placed pressure sensors. Nancy likes to roll over on her toes so we put sensors on the tops of her toes. She also likes to roll her head, so we placed two sensors on either side of her neck. For a duet piece, Elliott would do a backbend and in a hand balance, Inka would play on the sensors located on his hips with her toes.

\subsection{Sensor Design and Embedding}

We chose pressure and flex sensors to demonstrate weight and gravity for positional feedback as opposed to accelerometers which illustrate tilt.

We used Velostat (a electrically conductive packaging material made of polymeric foil impregnated with carbon black) and double-faced knitted conductive fabrics to make custom pressure sensors. We experimented with different shapes and fabrics, then tested pressure and flex sensors with various materials. We tested each sensor separately with a multimeter to get various flex levels. Then, integrating all of the sensors, calibrating for dynamic range of movement. We found the best design to be a 4" x 1" strip over the joint of the knee or elbow. A round design 3" x 3" worked well for the hip bones as they were more for partnering moves that involved pressure sensing.

We tried sewing the sensors first, but then realized that the contortionist would sweat during the performance and potentially short the circuit. To insure waterproof design we experimented encasing the sensors in plastic, then silicone and that lead to body painting them in place. This was the best option to keep them in specific body location and customize the fit. David had a body painting and sculpture background and knew of a body painting silicone for casting. He tested many styles of encasing and found painting the sensors and adding weights on top of them until they dried worked the best. After testing application techniques, we finally embedded the sensors in the silicone that was body painted on the artists as it was a more durable texture, custom fit, and waterproof. He decided to cover the contortionists with vaseline and body paint the silicone into a connected suit. This proved somewhat painful, as vaseline did not save their hair from being ripped out. In the end, they were such good sports and smooth skinned. 


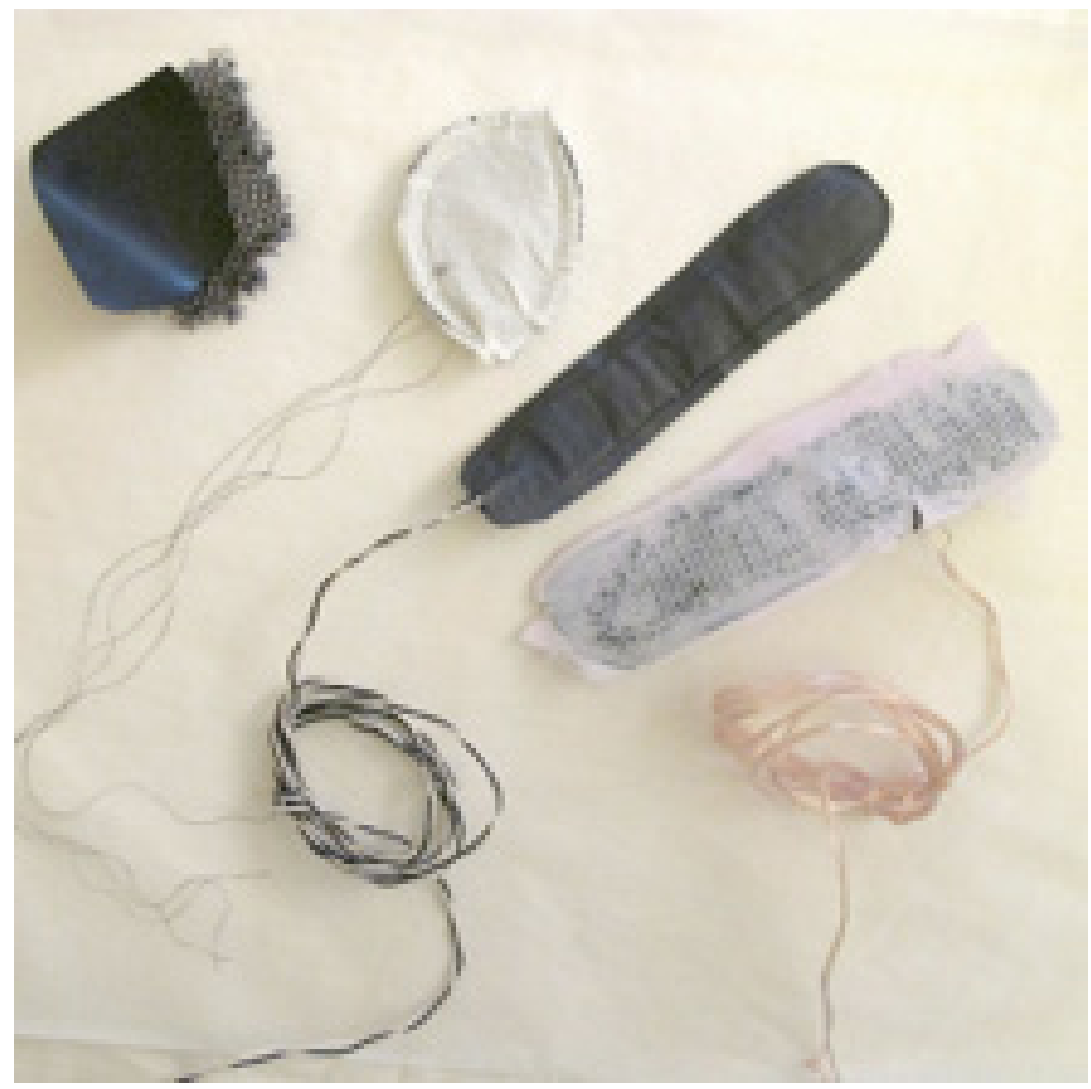

Fig. 2. Sensor development prototypes. From top left: the raw sensor materials, the sensors sewn, the sensors sealed, and the sensors fully encased in silicone.

Each suit had 11 sensors that would correspond to their featured solo and partner choreographies. We first mapped the sensors with movement. Then, we used multimeters to test the sensor resilience and shape. Once in the suit we had to adjust the resistance of each sensor within the range of movement.

Finally, we had wireless transmitters and Arduinos to communicate movement, and Processing program that created the music composed by a sound healing artist.

\subsection{Sound Design}

Initially, the sensors had varying degrees of flex create deeper tones. The more they move, the louder and deeper the tonal resonance. The sound artist created four sound-banks for the sensor placements that could be used at different times of the choreography. She also created a background track to support the soundscape. 
The healing sound artist, Stefanie $\mathrm{Ku}$, states:

Quantum Sound is our portrayal of the quantum world where everything is linked with such complexity that we cannot begin to consciously determine the cause and the effect. It is also an experiment in actualizing a turbo-charged version of synaesthesia, the ability to see sound or hear colors, to create new connections in the audience's minds with improbable pairings of action and sound.

We present to you via Quantum Sound an alternate world where the bending of an elbow or knee is accompanied by a modulated sample of a cheese grater in action, just as a Brazilian butterfly's fluttering wings inextricably causes a tempest in the middle the Mongolian desert.

The Quantum Sound sample library is constructed from heavily processed field recordings of seemingly mundane everyday sounds such as elevator mechanics and kitchen tools. The samples were put through a sonic blender of wave scanning synthesis, granular synthesis, and a collection of modulators (AM, FM and rotation), chopped and diced then reconstructed into "sonal characters" acting on stage with the contortionists as they exert various depth of pressure on the eleven sensors embedded strategically throughout their silicone suits.

\subsection{Integrated Suit}

It took experimentation to have the suits be elastic and strong enough to withstand extreme choreographies. After body painting the forms, we tried a few techniques to secure the silicone on the skin. We first tried medical tape, but it was not strong enough to hold in place. Then, we tried to adhere to a lycra body suit, but still needed more tension in specific vertexes. Eventually we found that series of silicone painted elastic bandages worked well for adding tensile strength.

The sensors are marked with phosphorescent pigment mixed in the silicone and UV lights illuminate when the sensor is flexed, adding visual feedback to the sound. They glow after being depressed. We decided to animate the wires that connect the sensors to the Arduino at the base of the neck to show that the body is plugged in. Finally, we had wireless Xbee radios to enable the communication of the suits to the laptop.

Amazingly, the wires did not tangle. We had a few silicone tears over the three performances, but we were able to fix these with silicone and pressure.

\section{Performance}

The performance was on a runway during an evening length fashion show. We began the performance with the two performers deliberately touching the sensors to show the fact that pressing a sensor creates a sound and a light. They began with solo tricks then mid-runway began partnering. The sound artist changed sound-banks with the 


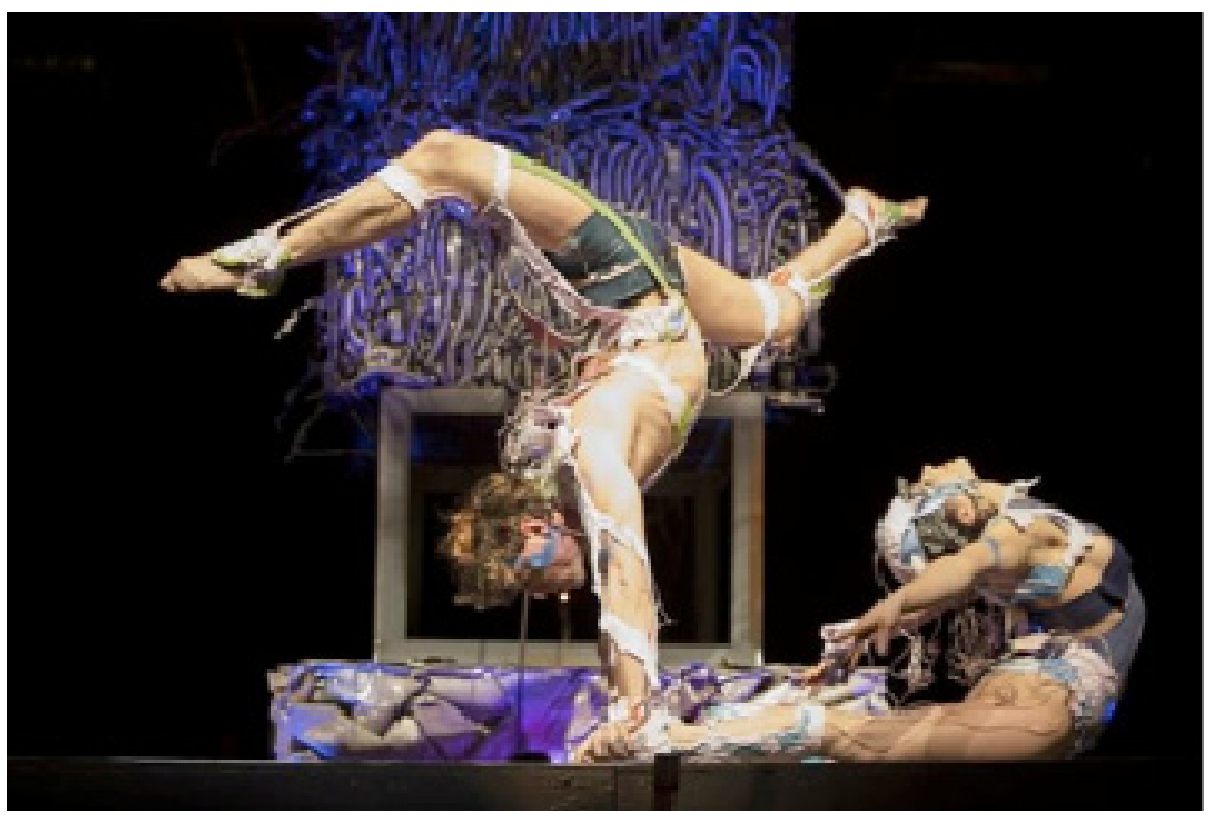

Fig. 3. The contortionists during performance

four sections of choreography - solos, duet, solos, grand finale duet. The collaborative team all unanimously decided which selections from the sound-bank were best for which section. For example, the duet section was more dynamic and energetic. The solo sections featured more subdued and introspective tones. The grand finale was an escalation of sounds as Elliott carried Inka off stage.

The painted silicone was drippy and David enhanced that look with green and copper for Elliott and blue and silver for Inka. Once in costume, the contortionists were inspired to be science fiction creatures from the cavernous electric swamp. Their movement was drippy and smooth and sometimes comedic.

During the performance, the once chatty crowd was silent and awestruck. As for the crowd reaction, some people did not realize the sound came from the suits. We decided in the future we should give a verbal introduction on stage before the performance to give context. Since it was a fashion show, they were not expecting technology.

Isolating specific sensors proved challenging; often a movement was joined by three sensors that would provide a harmony. It was easy for the contortionists to activate all the sensors at once, since their bodies were so strong and flexible.

\section{$5 \quad$ Reflections}

We learned that the suits help to animate the movement of the wearers. The performers felt like they could play their body like a keyboard and it was exciting for them to hear their sounds echoed in the room. They reported feeling larger, like their 
body filled the entire space. The sounds definitely enhanced movement as well as stillness to hush to sound and isolate specific tones.

We found that sound can also be a costume. Sound can outfit the body and to create tones with the body is empowering.

This design has potential to be used for yoga practice or even psychical therapy to tell proper body position. The tones could encourage and be incentives for movement. They could also limit movements or cause holding or balancing. The sound suit is a fun and engaging way to inhabit the body.

Acknowledgements. The authors would like to thank the project collaborators, Inka Siefker, Elliott Goodwin Gittelsohn, Stefanie Ku, Greg Ames, Anthony Ambuel, David Dawson, and Iris Beal for their amazing work and stellar performances.

\section{References}

1. Anderson, L.: Home of the Brave (video). Lumivision (1986)

2. Anderson, L.: Stories from the Nerve Bible: A Retrospective, pp. 1972-1992. Harper Perennial, New York (1994)

3. Coniglio, M.: MidiDancer (1989), http: //www.troikaranch.org/mididancer.html

4. Yamaha Miburui (1990), http: / / www.yamaha.co.jp/design/products/1990/miburi /

5. Paradiso, J.A., Eric, H.: Expressive footwear for computer-augmented dance performance. In: First International Symposium on Wearable Computers 1997. Digest of Papers, pp. 165-166. IEEE (1997)

6. Mulder, A.: Human Movement Tracking Technology. Report 94-1 of the Hand Centered Studies of Human Movement Project. Simon Fraser University, Burnaby (1994)

7. Winkler, T.: Making motion musical: Gesture mapping strategies for interactive computer music. In: ICMC Proceedings, pp. 261-264 (1995)

8. Siegel, W., Jacobsen, J.: The Challenges of Interactive Dance: An Overview and Case Study. Computer Music Journal 22(4), 29-43 (1998)

9. Rubin, S.: Crucible Fashion Show Lights it Up. SF Chronicle (January 7, 2013) 